\title{
Cross-order transfer of Wolbachia from Muscidifurax uniraptor (Hymenoptera: Pteromalidae) to Drosophila simulans (Diptera: Drosophilidae)
}

\author{
MARNIX M. M. VAN MEER* \& RICHARD STOUTHAMER \\ Agricultural University of Wageningen, Department of Plant Breeding and Crop Protection, Laboratory of \\ Entomology, Binnenhaven 7, 6709 PD Wageningen, The Netherlands
}

\begin{abstract}
Bacteria of the genus Wolbachia are widespread in arthropods and can induce different effects on the host such as cytoplasmic incompatibility (CI), thelytoky (T) or feminization (F). In some Wolbachia-infected hosts, no effect $(\mathrm{N})$ has been found. Successful transfer of Wolbachia by microinjection from one host to an uninfected one has been established with CI, F, $\mathrm{N}$-Wolbachia but not with T-Wolbachia. In this paper a transfer experiment of T-Wolbachia from the parasitoid Muscidifurax uniraptor to Drosophila simulans is described. The infection could be detected in the new host for several generations by polymerase chain reaction (PCR). However, no specific effects on the host were detected, and the bacteria were not stably maintained.
\end{abstract}

Keywords: cytoplasmic incompatibility, Drosophila, microinjection, Muscidifurax, thelytoky, Wolbachia.

\section{Introduction}

Intracellular bacteria of the genus Wolbachia (Rickettsiales) are found in many different insect species. Although infection with these bacteria was known from mosquitoes at the beginning of this century (Hertig, 1936), it was only with the availability of polymerase chain reaction (PCR) technology at the end of the 1980s that the widespread occurrence of these bacteria was revealed. Screening of insect species from different orders from Panama revealed an infection rate of 16\% (Werren et al., 1995a).

Wolbachia are cytoplasmically inherited and can have a number of different effects on their hosts: (i) cytoplasmic incompatibility $(\mathrm{CI})$, which is a crossing incompatibility in which sperm from Wolbachiainfected males cannot successfully fertilize eggs from uninfected females, whereas all other crosses are fertile (Yen \& Barr, 1971; Trpis et al., 1981; Noda, 1984; Wade \& Stevens, 1985; Hoffmann et al., 1986; Breeuwer \& Werren, 1990); (ii) thelytoky (T), which is found in Hymenoptera - unfertilized eggs from Wolbachia-infected females develop into females, whereas they would normally develop into males

*Correspondence. E-mail: marnix.vanmeer@medew.ento.wau.nl.
(Stouthamer et al., 1990, 1993; Zchori-Fein et al., 1995); (iii) feminization (F), which has only been found in isopods (Crustacea) in which the infection causes genetic males to reproduce as females (Juchault et al., 1994); (iv) no apparent effect (N) on the reproduction of infected individuals at all, and this has been found in several Drosophila species (Giordano et al., 1995; Hoffmann et al., 1996).

The phylogeny of Wolbachia has been studied extensively based on several Wolbachia genes: 16S rDNA (O'Neill et al., 1992; Rousset et al., 1992; Stouthamer et al., 1993) and the cell cycle gene fts $Z$ (Holden et al., 1993; Werren et al., 1995b). These studies show that two main groups of sequences can be distinguished and these have been named group A and group B (Werren et al., 1995b). However, it appears that Wolbachia inducing similar effects on hosts are not monophyletic and are present in both groups. Very closely related Wolbachia can have quite different effects on their host. This absence of an association between monophyly and the different effects on the host has led to several hypotheses. One hypothesis is that a non-specific Wolbachia strain induces a distinct host response, e.g. a Wolbachia strain inducing CI in one host would induce thelytoky in a hymenopteran host, but so far this has 
not been found. Alternative hypotheses for the lack of congruence between effect on host and phylogeny are: (i) Wolbachia genes coding for a certain effect ( T, CI or F) are located on a plasmid that can jump relatively easily from one Wolbachia strain to another; (ii) Wolbachia genes inducing T, CI or F can cross over between different strains in doubleinfected hosts; (iii) Wolbachia genes causing T, CI or $\mathrm{F}$ are highly congruent and can readily evolve from each other.

One method to determine whether (i) a specific Wolbachia strain causes a distinct effect on the host or (ii) different hosts experience different effects with the same Wolbachia strain is to create new combinations of host and symbiont. Such transfer experiments have been carried out successfully with CI-Wolbachia among several Drosophila spp. both intraspecifically (Rousset \& De Stordeur, 1994) and interspecifically (Boyle et al., 1993; Clancy \& Hoffmann, 1997) and interspecifically in Tribolium confusum (Chang \& Wade, 1994). In addition, interfamily transfer of CI-Wolbachia has been realized. Wolbachia from the mosquito Aedes albopictus were transferred to the fruitfly $D$. simulans, and this revealed that the transinfected Drosophila strain became bidirectionally incompatible with all other naturally infected strains (Braig et al., 1994). F-Wolbachia causing feminization have also been transferred inter- and intraspecifically in several species of isopods (Juchault et al., 1994) and induced similar effects in their hosts. Finally, N-Wolbachia causing no obvious effect on the host were transferred from $D$. mauritiana to $D$. simulans in which they also failed to cause any obvious effect (Giordano et al., 1995). From these studies, it became clear that similar effects were found in the new host-Wolbachia combination as in the original one.

No such transfer experiments have been performed with T-Wolbachia. Here, we report on experiments we have conducted to determine whether T-Wolbachia found in the hymenopteran
Muscidifurax uniraptor would cause a specific effect in the dipteran host Drosophila simulans. It was determined whether injection of T-Wolbachia induced mortality in $D$. simulans eggs and if the infection would become established in the new host. Secondly, when the infection succeeded, effects on the host were studied by: (i) measuring egg mortality of infected eggs; and (ii) performing crosses to investigate whether T-Wolbachia were able to induce CI in its new host. CI-Wolbachia were also injected in uninfected $D$. simulans to verify the microinjection protocol.

\section{Materials and methods}

\section{Insect strains}

The insect and Wolbachia strains used in the injection experiment are shown in Table 1.

Flies were maintained in bottles containing Drosophila medium [ $20 \mathrm{~g}$ of agar, $135 \mathrm{~g}$ of sugar, $38 \mathrm{~g}$ of yeast, $0.12 \mathrm{~g}$ of nipagine $\left(10 \mathrm{~g} \mathrm{~L}^{-1}\right.$ nipagine in $70 \%$ ethanol) made up in $1 \mathrm{~L}$ of water] and incubated at $20^{\circ} \mathrm{C}$. The fly pupa parasitoid $M$. uniraptor originated from Puerto Rico (Legner, 1985) and was reared on Musca domestica pupae and incubated at $25^{\circ} \mathrm{C}$.

\section{Microinjection}

Microinjection was performed according to the method of Santamaria (1986) and Boyle et al. (1993) with some modifications. Embryos were collected over a 30-min period, transferred to double-sided sticky tape on a slide, dechorionated manually and transferred to a new slide. The embryos were partially desiccated by exposure to the air for up to $4 \mathrm{~min}$ (depending on the relative humidity) and subsequently covered with heavy mineral oil (Sigma, no. 400-5). Using a microcapillary needle (Boehringer femtotips), ooplasm containing Wolbachia ( $w$ Uni or $w \mathrm{Ri}$ ) was drawn from infected eggs and then injected into the posterior poles of unin-

Table 1 Insect species and Wolbachia strains used in the microinjection experiment

\begin{tabular}{lcccc}
\hline Species & $\begin{array}{c}\text { Wolbachia } \\
\text { strain } \\
\text { abbreviation }\end{array}$ & $\begin{array}{c}\text { Effect } \\
\text { in host }\end{array}$ & $\begin{array}{c}\text { Abbreviation for new } \\
\text { transinfected } \\
\text { species }\end{array}$ \\
\hline Drosophila simulans Riverside & $\mathrm{D}$ & $w \mathrm{Ri}$ & $\mathrm{CI}$ & - \\
Muscidifurax uniraptor & $\mathrm{D}$ & WUni & $\mathrm{T}$ & - \\
Drosophila simulans Watsonville & $\mathrm{A}$ & Injected with $w \mathrm{Ri}$ & $\mathrm{CI}$ & $\mathrm{DSW}(\mathrm{CI})$ \\
Drosophila simulans Watsonville & $\mathrm{A}$ & Injected with $w$ Uni & $?$ & $\mathrm{DSW}(\mathrm{T})$ \\
\hline
\end{tabular}

Ooplasm was isolated from the donor species (D) and injected into the acceptor (A). 
fected $D$. simulans Watsonville embryos before pole cell formation. Injected embryos were incubated overnight at $25^{\circ} \mathrm{C}(18 \mathrm{~h})$. On the following day, hatching larvae were isolated at 2-h intervals and transferred to vials with Drosophila medium. After pupation and eclosion, isofemale lines were started. Adult virgin females were crossed with uninfected males, because some males from the injected eggs failed to mate or were sterile. A sample of the injected eggs was used to measure the mortality of eggs, larvae and pupae.

\section{Rearing of isofemale lines}

In the first generation after injection, all females were tested for Wolbachia infection. From the offspring of each of these isofemale lines, five new isofemale lines were started. After 1 week, these five females were tested for Wolbachia infection. Offspring from infected mothers were used to start new isofemale lines. In the $3 \mathrm{rd}-7$ th generations, new DSW(T) lines were established from females that had the highest proportion of infected offspring. Adult transinfected females were tested for Wolbachia infection by PCR amplification of the fts $Z$ gene (Holden et al., 1993).

\section{Egg mortality, fecundity and incompatibility testing}

To investigate whether $w$ Uni infection in DSW(T) caused egg mortality, the number of hatched eggs of infected DSW(T) females was counted. Females were taken from an infected DSW(T) line and the uninfected DSW line (control). They were placed on agar dishes covered with fresh yeast. After $48 \mathrm{~h}$, these females were placed on a new agar dish with yeast. When the agar dishes had been incubated at $20^{\circ} \mathrm{C}$ for $48 \mathrm{~h}$, the numbers of eggs and larvae were counted. At the end of the experiment, females were prepared for PCR to check for Wolbachia infection. Data from uninfected females from the DSW(T) line, according to PCR, were removed before analysis of the results. Experiments were performed in duplicate. The numbers of eggs from infected and non-infected mothers were also compared.

Egg mortality was used to indicate reproductive incompatibility. To test whether $w$ Uni induced CI in $\operatorname{DSW}(\mathrm{T})$, matings between transinfected males and uninfected females were set up. For each cross, 201 to 2-day-old DSW virgin females were each allowed to mate with one 5-day-old $\operatorname{DSW}(\mathrm{T})$ male in the second generation or one 1-day-old male in the fourth generation. As a control, 10 virgin DSW females (from the same batch as above) were crossed with uninfected DSW males. To confirm the unmated status of these females, a control vial of 10 virgin females was taken. All DSW females were checked for Wolbachia infection to confirm their uninfected status. After $24 \mathrm{~h}$, males were removed and tested for Wolbachia infection. Females were allowed to lay eggs for 2 days on agar covered with fresh yeast. After removing the females, the dishes were incubated for $48 \mathrm{~h}$, and the numbers of eggs and larvae were counted. Experiments were performed in duplicate, and the mortality rates were compared with a Kruskal-Wallis test.

\section{DNA extraction and PCR}

Drosophila flies were homogenized with a clean pestle in $100 \mu \mathrm{L}$ of STE $(100 \mathrm{~mm} \mathrm{NaCl}, 10 \mathrm{~mm}$ Tris, 1 mм EDTA, pH 8.0) (O’Neill et al., 1992) and incubated with $2 \mu \mathrm{L}$ of Proteinase $\mathrm{K}\left(20 \mathrm{mg} \mathrm{mL}^{-1}\right)$ for $1 \mathrm{~h}$ at $37^{\circ} \mathrm{C}$, followed by $5 \mathrm{~min}$ at $95^{\circ} \mathrm{C}$. Samples were spun for $2 \mathrm{~min}$ in a centrifuge, and $1 \mu \mathrm{L}$ of the supernatant was used as template in subsequent PCR reactions. The Wolbachia fts $Z$ gene was amplified using specific fts $Z$ primers (Holden et al., 1993): 5'-GGACCGGATCCGTATGCCGATTGCAGAGCTTG-3' and 5'-GGACCGAATTCGCCATGAGTATTCACTTGGCT-3'. For PCR, a temperature profile of $94^{\circ} \mathrm{C}$ for $2 \mathrm{~min}$ ( 1 cycle); $94^{\circ} \mathrm{C}$ for $1 \mathrm{~min}$; $55^{\circ} \mathrm{C}$ for $1 \mathrm{~min} ; 72^{\circ} \mathrm{C}$ for $1 \mathrm{~min}$ (40 cycles) was used.

Confirmation of the identity of the Wolbachia strain in the infected Drosophila host

Fts $Z$ sequences of Wolbachia from D. simulans Riverside $w \mathrm{Ri}$ and $M$. uniraptor $w$ Uni were retrieved from GenBank (accession numbers U28178 and U28186) and analysed for differences (Werren et al., 1995b). The fts $Z$ primers (Holden et al., 1993) amplify the region $494-1221 \mathrm{bp}$ of the fts $Z$ gene, which includes a part of the 3'flanking sequence. The $792 \mathrm{bp}$ sequences (including the primers) differ in four nucleotides, and $P v u$ II cuts at position 1120 of the $w$ Ri fts $Z$ sequence. PvuII restriction digestion results in two fragments of $659 \mathrm{bp}$ and $133 \mathrm{bp}$ in the case of the fts $Z$ of $w \mathrm{Ri}$ Wolbachia.

\section{Results}

Mortality rates of injected eggs

Mortality rate differences were found between eggs injected with Drosophila cytoplasm containing $w \mathrm{Ri}$ or Muscidifurax cytoplasm containing $w$ Uni (Table 2). Eggs injected with $w$ Uni showed higher 
Table 2 Mortality rates of different life stages of Drosophila simulans Watsonville injected with different cytoplasm types and Wolbachia strains

\begin{tabular}{|c|c|c|c|c|c|c|}
\hline \multirow[b]{2}{*}{ Injected } & \multicolumn{3}{|c|}{ Egg to larva } & \multicolumn{3}{|c|}{ Larva to pupa } \\
\hline & Alive & Dead & Total & Alive & Dead & Total \\
\hline$w R i$ & 98 & 142 & 240 & 58 & 40 & 98 \\
\hline \multirow[t]{2}{*}{$w$ Uni } & 140 & 75 & 215 & 66 & 74 & 140 \\
\hline & \multicolumn{3}{|c|}{ Larva to adult } & \multicolumn{3}{|c|}{ Pupa to adult } \\
\hline Injected & Alive & Dead & Total & Alive & Dead & Total \\
\hline$w \mathrm{Ri}$ & 48 & 50 & 98 & 48 & 10 & 58 \\
\hline$w$ Uni & 52 & 88 & 140 & 52 & 14 & 66 \\
\hline \multicolumn{7}{|l|}{ Adults } \\
\hline Injected & Total females & $\begin{array}{l}\text { Total females } \\
\text { with offspring }\end{array}$ & Total males & $\begin{array}{c}\text { Total } \\
\text { males + females }\end{array}$ & & \\
\hline$w \mathrm{Ri}$ & 22 & 11 & 26 & 48 & & \\
\hline$w$ Uni & 25 & 11 & 27 & 52 & & \\
\hline
\end{tabular}

hatching rates than eggs injected with $w \mathrm{Ri}$. However, this difference is not seen when mortality rates of larvae or pupae are compared. Of the resulting adult females, approximately $50 \%$ produced offspring.

\section{Wolbachia infection rates}

One positive DSW(CI) line was found from 11 lines injected with $w \mathrm{Ri}$. From this infected mother, all offspring were infected (five individuals tested per generation) in the third and consecutive generations. The infection remained stable for at least 10 generations. Of 41 isofemale lines injected with $w$ Uni, 10 lines had infected offspring rates ranging from $20 \%$ to $80 \%$. The percentage infected offspring of infected mothers decreased to $60 \%$ at maximum in successive generations, and no infected offspring were found in the seventh and consecutive generations (Table 3). PCR with the fts $Z$ primers was performed on $D$. simulans Watsonville (DSW) and Riverside, M. uniraptor and the transinfected lines $\operatorname{DSW}(\mathrm{CI})$ and DSW(T). No product was amplified from DSW, whereas all other samples resulted in a PCR product of the expected size (792 bp). Restriction analysis on these fts $Z$ products confirmed the identity of the $w$ Uni strain in $D$. simulans (data not shown).
Effects on host resulting from wUni infection in D. simulans

In the second generation after injection, effects on the host caused by the $w$ Uni infection in DSW(T) were studied by determining female fecundity and egg mortality rates from infected mothers. No differences were found between infected and DSW mothers (Table 4). Secondly, expression of CI by infected $w$ Uni males was studied. If $w$ Uni induces CI in its host, reduced egg hatching rates should be found in the crossing of DSW females $\times$ DSW $(T)$ males. But no reduced egg hatching rates were found in the putative incompatible crossing in comparison with the control crossing (Table 4).

In the fourth generation, similar egg mortality results were obtained from the putative $\mathrm{CI}$ and control crossing (Table 4). Egg mortality from DSW(T) mothers was determined in the fourth generation as well but, because of the low transmission rate (26\%; Table 3), insufficient positive samples were obtained.

\section{Discussion}

It was not known whether the injection of foreign cytoplasm into $D$. simulans eggs would result in higher mortality. Because of the different microin-

(C) The Genetical Society of Great Britain, Heredity, 82, 163-169. 
jection conditions between the actual experiments, our experimental set-up is not suitable for addressing this issue. However, comparing the mortality in both experiments, it becomes clear that Drosophila eggs can survive the introduction of foreign cytoplasm (Table 2). In addition, $w$ Uni injection does not result in excessive mortality in the different developmental stages of Drosophila.
Wolbachia could be detected by PCR in the DSW(CI) and DSW(T) lines in the second generation after injection. The transmission was $100 \%$ in the second generation for DSW(CI) and was stable for at least 10 generations. For DSW(T), however, the highest transmission rate was found in the second generation $(80 \%)$ and decreased to $0 \%$ in the seventh generation.

Table 3 Number of isofemale lines of Drosophila simulans tested, number of individuals tested per line and offspring infection rates from 2 nd to 10 th generations of DSW(T) injected females

\begin{tabular}{lccc}
\hline $\begin{array}{l}\text { Generation } \\
\text { after injection }\end{array}$ & $\begin{array}{c}\text { No. of isofemale } \\
\text { lines tested }\end{array}$ & $\begin{array}{c}\text { No. of individuals } \\
\text { per isofemale line }\end{array}$ & $\begin{array}{c}\text { \% infected } \\
\text { offspring }\end{array}$ \\
\hline 2 & 31 & 5 & 0 \\
& 3 & 10 & 20 \\
& 3 & 5 & 40 \\
& $1^{*}$ & 40 & 47.5 \\
3 & 1 & 5 & 60 \\
& 2 & 5 & 80 \\
4 & 1 & 5 & 0 \\
& 1 & 5 & 20 \\
5 & 3 & 5 & 60 \\
& $1 *$ & 38 & 26 \\
6 & 3 & 10 & 40 \\
7 & 2 & 5 & 0 \\
$8,9,10$ & 1 & 5 & 20 \\
& 3 & 5 & 60 \\
& 2 & 10 & 0 \\
\end{tabular}

*Individuals from these isofemale lines were used in egg mortality and CI experiments.

Table 4 Number of eggs with standard error (SE) and mean egg mortality with standard deviation (SD) from different mating combinations between uninfected Drosophila simulans (DSW) and $w$ Uni-infected DSW(T), two and four generations after injection (crosses $\mathrm{b}$ and $\mathrm{f}$ represent the putative CI crossing)

\begin{tabular}{llccc}
\hline $\begin{array}{l}\text { Generation tested } \\
\text { (after infection) }\end{array}$ & Cross (female $\times$ male) & $\begin{array}{c}\text { Eggs/female } \\
\pm \text { SE (number } \\
\text { of females tested) }\end{array}$ & $\begin{array}{c}\text { Mean egg } \\
\text { mortality } \\
\pm \text { SD }\end{array}$ & $\begin{array}{l}\text { Egg mortality } \\
\text { comparison }\end{array}$ \\
\hline 2 & a: DSW $\times$ DSW & $79 \pm 6(10)$ & $12.7 \pm 7$ & a vs. b $P=0.42$ \\
& b: DSW $\times$ DSW(T) & $86 \pm 8(12)$ & $10.3 \pm 10$ & \\
& c: DSW $\times$ DSW & $64 \pm 7(9)$ & $4.8 \pm 5$ & c vs. d $P=0.64$ \\
d: DSW(T) $\times$ DSW(T) & $78 \pm 9(9)$ & $3.6 \pm 5$ & e vs. f $P=0.09$ \\
& e: DSW $\times$ DSW & $59 \pm 5(10)$ & $8.4 \pm 5$ & \\
\hline
\end{tabular}


Effects on the host were studied for DSW(T) in the second and fourth generations. Because D. simulans is not adapted to thelytoky, egg mortality was expected to occur. But no significantly higher mortality was found in eggs from infected mothers in the second generation, which corresponded with the decreasing transmission rates. In addition, no fecundity differences could be found between infected and non-infected females. Because Wolbachia can induce CI in Drosophila (Hoffman et al., 1986), this phenomenon was tested for with the w Uni transinfected Drosophila strains. No CI could be detected in the second and fourth generations after injection in the incompatible crosses. In other cytoplasm injection experiments, Wolbachia effects in the host could be detected as early as two generations after injection (Boyle et al., 1993). In the injection experiment of Braig et al. (1994), Wolbachia could only be detected by PCR after six generations. But the set-up of this experiment was different because purified Wolbachia were injected, which could have an effect on Wolbachia fitness or result in a difference in injected Wolbachia dose.

Although the $f t s Z$ sequences of $w \mathrm{Ri}$ and $w \mathrm{Uni}$ are closely related $(0.4 \%$ difference), no permanent establishment of Wolbachia from M. uniraptor infection occurred in D. simulans. Because $w$ Uni infection could be detected for several generations, Wolbachia had to be present in the eggs. However, no effects on the host could be detected, and transmission rates decreased after each generation. Because no correlation was found between the decreased transmission rates and egg mortality, it remains unclear why the infection is not stable in its new host. One explanation might be that, when no CI mechanism is present, this being the driving force for the spread of Wolbachia through the population (Turelli, 1994), Wolbachia could disappear from the population. However, transfer experiments with $\mathrm{N}-$ Wolbachia revealed that the new hosts had $100 \%$ infected offspring as well, indicating that no CI mechanism was necessary for their persistence (Giordano et al., 1995). A second explanation for the infection not being stable is that $w$ Uni is not physiologically adapted to its new host. Consequently, the Wolbachia density would not rise sufficiently, explaining the fact that no effects were observed on the host (Boyle et al., 1993; Breeuwer \& Werren, 1993). As found in D. serrata, a novel host for Wolbachia, Wolbachia from D. simulans Riverside were successfully transferred to this species, but maternal transmission was relatively low (Clancy \& Hoffmann, 1997), indicating that host-symbiont interactions are important for success in the estab- lishment of the infection. An example of Wolbachia transmission failure was found in isopods. Injection experiments revealed that Wolbachia could be transferred between closely related species of isopods (Armadillidium nasatum to A. vulgare). However, transmission of Wolbachia from Chaetophiloscia elongata to $A$. vulgare, which are more distantly related species, failed (Juchault et al., 1994). This might be a similar case in which Wolbachia were not adapted to their new host. On the other hand, phylogenetic work on Wolbachia (Werren et al., 1995b) provides evidence that, in some cases, this bacterium has successfully bridged large phylogenetic distances in its interspecific movement, and it remains unknown what permits particular interorder transfers to occur successfully. When practical microinjection problems are overcome, future experiments could reveal whether $w$ Uni can be transferred to other hymenopteran species and if similar phenotypes are induced as in the original host.

\section{Acknow ledgements}

We thank D. Poinsot for providing D. simulans Watsonville and Riverside strains. We also thank J. Honda, I. Silva, K. Flanagan and F. van Kan for technical support. Finally, we thank C. Stolk, S. Sinkins, the Ph.D. students of our Laboratory of Entomology and two anonymous reviewers for giving critical comments on this manuscript. This project was funded by the Technology Foundation (STW), grant number 790.42.793, of The Netherlands.

\section{References}

BOYLE, L., O'NEILL, S. L., ROBERTSON, H. M. AND KARR, T. L. 1993. Interspecific and intraspecific horizontal transfer of Wolbachia in Drosophila. Science, 260, 1796-1799.

BRAIG, H. R., GUZMAN, H., TESH, R. B. AND O'NEILL, S. L. 1994. Replacement of the natural Wolbachia symbiont of Drosophila simulans with a mosquito counterpart. Nature, 367, 453-455.

BREEUWER, J. A. J. AND WERrEN, J. H. 1990. Microorganisms associated with chromosome destruction and reproductive isolation between two insect species. Nature, 346, 558-560.

BREEUWER, J. A. J. AND WERREN, J. H. 1993. Cytoplasmic incompatibility and bacterial density in Nasonia vitripennis. Genetics, 135, 565-574.

CHANG, N. W. AND WADE, M. J. 1994. The transfer of Wolbachia pipientis and reproductive incompatibility between infected and uninfected strains of the flour beetle Tribolium confusum by microinjection. Can. J. Microbiol., 40, 978-981.

(C) The Genetical Society of Great Britain, Heredity, 82, 163-169. 
ClANCY, D. J. AND HOFFMANN, A. A. 1997. Behavior of Wolbachia endosymbionts from Drosophila simulans in Drosophila serrata, a novel host. Am. Nat., 149, 975-988.

GIORDANO, R., O'NEILl, S. L. AND ROBERTSON, H. M. 1995. Wolbachia infections and the expression of cytoplasmic incompatibility in Drosophila sechellia and D. mauritiana. Genetics, 140, 1307-1317.

Hertig, M. 1936. The Rickettsia, Wolbachia pipientis and associated inclusions of the mosquito, Culex pipiens. Parasitology, 28, 453-490.

HOFFMANN, A. A., TURELli, M. AND SIMMONS, G. M. 1986. Unidirectional incompatibility between populations of Drosophila simulans. Evolution, 40, 692-701.

HOFFMANN, A. A., ClANCY, D. J. AND DUNCAN, J. 1996. Naturally-occurring Wolbachia infection in Drosophila simulans that does not cause cytoplasmic incompatibility. Heredity, 76, 1-8.

HOLDEN, P. R., BROOKFIELD, J. F. AND JONES, P. 1993. Cloning and characterization of an fts $Z$ homologue from a bacterial symbiont of Drosophila melanogaster. Mol. Gen. Genet., 240, 213-220.

JUChAUlt, P., FRELON, M., BOUCHON, D. AND RIGAUD, T. 1994. New evidence for feminizing bacteria in terrestrial isopods. C.R. Acad. Sci. Paris, 317, 225-230.

LEGNER, E. F. 1985. Effects of scheduled high temperature on male production in thelytokous Muscidifurax uniraptor. Can. Ent., 117, 383-389.

NODA, H. 1984. Cytoplasmic incompatibility in allopatric field populations of the small brown planthopper, Laodelphax striatellus in Japan. Entomologia Exp. Appl., 35, 263-267.

O'NEILL, S. L., GIORDANO, R., COLBERT, A. M. E., KARR, T. L. AND ROBERTSON, H. M. 1992. 16S rRNA phylogenetic analysis of the bacterial endosymbionts associated with cytoplasmic incompatibility in insects. Proc. Natl. Acad. Sci. U.S.A., 89, 2699-2702.

ROUSSET, F. AND DE STORDEUR, E. 1994. Properties of Drosophila simulans strains experimentally infected by different strains of the bacterium Wolbachia. Heredity, 72, 325-331.

ROUSSET, F., BOUChON, D., PINTUREAU, B., JUCHAUlT, P. AND SOLIGNAC, M. 1992. Wolbachia endosymbionts responsible for various alterations of sexuality in arthropods. Proc. R. Soc. B, 250, 91-98.

SANTAMARIA, P. 1986. Injecting eggs. In: Roberts., D. B. (ed.) Drosophila: a Practical Approach, pp. 159-173. IRL Press: Oxford.

STOUTHAMER, R., LUCK, R. F. AND HAMILTON, W. D. 1990. Antibiotics cause parthenogenetic Trichogramma (Hymenoptera/Trichogrammatidae) to revert to sex. Proc. Natl. Acad. Sci. U.S.A., 87, 2424-2427.

STOUTHAMER, R., BREeUWer, J. A. J., LUCK, R. F. AND WERREN, J. H. 1993. Molecular identification of microorganisms associated with parthenogenesis. Nature, 361, 66-68.

TrPis, M., PERrone, J. B., REISSIG, M. AND PARKER, K. L. 1981. Control of cytoplasmic incompatibility in the Aedes scuttelaris complex. J. Hered., 72, 313-317.

TURELLI, M. 1994. Evolution of incompatibility-inducing microbes and their hosts. Evolution, 48, 1500-1513.

WADE, M. J. AND STEVENS, L. 1985. Microorganism mediated reproductive isolation in flour beetles (genus Tribolium). Science, 227, 527-528.

WERREN, J. H., GUO, L. R. AND WINDSOR, D. W. 1995a. Distribution of Wolbachia in neotropical arthropods. Proc. R. Soc. B, 262, 197-204.

WERrEN, J. H., ZHANG, W. AND GUO, L. R. 1995b. Evolution and phylogeny of Wolbachia: reproductive parasites of arthropods. Proc. R. Soc. B, 251, 55-63.

YEN, J. H. AND BARR, A. R. 1971. New hypothesis of the cause of cytoplasmic incompatibility in Culex pipiens. Nature, 232, 657-658.

ZCHORI-FEIN, E., FAKTOR, O., ZEIDAN, M., GOTTLIEB, Y., CZOSNEK, H. AND ROSEN, D. 1995. Parthenogenesisinducing microorganisms in Aphytis. Insect Mol. Biol., 4, 173-178. 